\title{
A review: potential of resveratrol and its analogues to mitigate diseases via gut microbial modulation
}

\author{
Yen-Chun Koh ${ }^{\mathrm{a}}$, You-Tsz Lien ${ }^{\mathrm{a}}$, Yu-Ting Chou ${ }^{\mathrm{b}}$, Chi-Tang $\mathrm{Ho}^{\mathrm{c}}$ \\ and Min-Hsiung Pan a,d,e*
}

\begin{abstract}
anstitute of Food Sciences and Technology, National Taiwan University, Taipei, Taiwan
${ }^{b}$ Department of Food Science, National Taiwan Ocean University, Keelung, Taiwan

'Department of Food Science, Rutgers University, New Brunswick, New Jersey, USA

${ }^{\mathrm{d} D e p a r t m e n t}$ of Medical Research, China Medical University Hospital, China Medical University, Taichung City, Taiwan

e Department of Health and Nutrition Biotechnology, Asia University, Taichung City, Taiwan

*Corresponding author: Min-Hsiung Pan, Institute of Food Science and Technology, National Taiwan University, No. 1, Section 4, Roosevelt Road, Taipei 10617, Taiwan. Tel: +886-2-33664133; Fax:+886-2-33661771; E-mail: mhpan@ntu.edu.tw
\end{abstract}

DOI: $10.31665 /$ JFB.2020.12248

Received: December 12, 2020; Revised received \& accepted: December 28, 2020

Citation: Koh, Y.-C., Lien, Y.-T., Chou, Y.-T., Ho, C.-T., and Pan, M.-H. (2020). A review: potential of resveratrol and its analogues to mitigate diseases via gut microbial modulation. J. Food Bioact. 12: 97-105.

\begin{abstract}
The stilbenoids form a group of bioactive phenolic compounds found in various plant species whose original functions are to act as protective compounds against microbial infections and toxins. Among the stilbenoids, resveratrol is the most well-studied and has been shown to exert multiple benefits in disease prevention. The bioavailability of resveratrol is one of the major limitations in its role as a disease-prevention agent; however, it has exhibited biological activity in animals and clinical models. Gut microbiota may play a role in overcoming limited bioavailability via microbial transformations. On the other hand, the modulatory effects of stilbenoids on gut microbial dysbiosis induced by several diseases can be crucial in disease alleviation. In addition to gut microbial metabolites, such as SCFAs (short-chain fatty acids), stilbenoids can be used to inhibit microbial growth, making their use a potential strategy in preventing disease progression. In this review, the interactions of stilbenoids (with a major focus on resveratrol and pterostilbene) and gut microbiota will be discussed to clarify the importance of gut microbiota in the strategy of "disease prevention via phytochemicals."
\end{abstract}

Keywords: Stilbenoids; Resveratrol; Pterostilbene; Gut microbiota.

\section{Introduction}

The term "bioavailability" defines the potential and percentage of the administered substance, i.e., the uptake dose of the substance, be it a drug or xenobiotic, that finally reaches the circulation system of a living organism (Nikinmaa, 2014). Generally, the bioavailability of a substance administrated intravenously is practically $100 \%(\mathrm{~F}=1)$, while those substances administered orally or through dietary intake may have much lower bioavailability $(\mathrm{F}<$ 1) due to several issues, including incomplete absorption, polarity of the substances, and the possibility of the target substance be- ing metabolized (known as the first-pass effect) (Hinderliter and Saghir, 2014; Waller and Sampson, 2018). Bioavailability is one of the major concerns when considering dietary phytochemicals as strategies for achieving health benefits. However, phytochemicals are normally transient due to their low bioavailability, meaning that the concentration of the parent compound could be low in the circulatory system (Selby-Pham et al., 2017). Consumption of phytochemicals in the diet can reach up to several grams, but the concentration of the final fraction absorbed and circulated in body may be much lower than expected (Martel et al., 2020). Therefore, the efficacy of phytochemicals in the amelioration of 
<smiles>Oc1ccc(/C=C/c2cc(O)cc(O)c2)cc1</smiles>

b<smiles>COc1cc(O)cc(/C=C/c2ccc(O)cc2)c1</smiles>

C<smiles>COc1cc(/C=C/c2ccc(O)cc2)cc(OC)c1</smiles>

d<smiles>Oc1cc(O)cc(/C=C/c2ccc(O)cc2O)c1</smiles>

e<smiles>Oc1cc(O)cc(C=Cc2ccc(O)c(O)c2)c1</smiles><smiles>COc1cc(O)cc(/C=C/c2cccc(O)c2)c1</smiles>

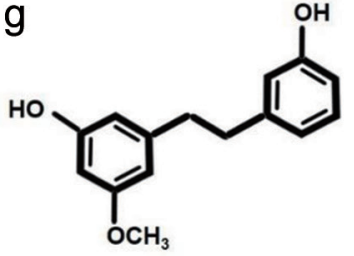

Figure 1. Resveratrol and its analogues found naturally in plants. (a) Resveratrol, (b) pinostilbene, (c) pterostilbene, (d) oxyresveratrol, (e) piceatannol, (f) thunalbene, and (g) batatasin III.

several diseases could be the result of the phytochemical itself or its derivatives, metabolites produced in the liver or colon tissue, or microbial metabolites.

According to the latest definition, polyphenols can be categorized as prebiotic-like components via their influence on gut microbiota, including the stimulation of beneficial gut microbes, which leads to beneficial microbial metabolite production, balances the gut ecosystem, and protects the organism from pathogens, resulting in host health benefits (Peng et al., 2020). The beneficial outcomes for gut microflora under optimal conditions may include lowering blood ammonia, modulating immune responses, and reducing cancer risks (Manning and Gibson, 2004). The relationship between gut microbiota and phytochemicals, such as polyphenols, permits substituting conjugates, hydrolysis, reduction, and cleavage or degradation; therefore, the presence of metabolites in plasma can be extended beyond that of the parent compounds (Carrera-Quintanar et al., 2018). It is possible that the catabolism of polyphenols by gut microbes could release metabolites or derivatives with better bioactive effects and absorption (Simó and García-Cañas, 2020). Intervention via phytochemicals may produce multi-benefits for gut health, including: (1) enrichment of beneficial bacteria, (2) inhibited growth or reduced levels of opportunistic bacteria, (3) facilitated growth of short-chain fatty acid (SCFA) producers that metabolize bile acids, (4) inhibited digestive enzymes, and (5) enhanced colonic tight junctions (Martel et al., 2020). In this review, we focus on the regulatory effects of resveratrol and pterostilbene on gut microbiota and the potential benefits for the host in terms of health and disease prevention.

\section{Resveratrol potentially interacts with gut microbiota to im- prove host health}

The benefits of resveratrol (Figure 1) with regard to gut health has been recently revealed by Chen et al., who showed that resveratrol plays multiple roles, including enhancing the intestinal barrier (upregulating occludin, ZO1, claudin1), inhibiting inflammation, and attenuating gut microbial dysbiosis (Chen et al., 2020). Among the gut microbes, Akkermansia muciniphila, Ruminococcaceae, and Lachnospiraceae were enhanced, while Desulfovibrio growth was inhibited. In an in vitro study using M-SHIME ${ }^{\circledR}$ to investigate the changes in the gut microbiota of a female volunteer, it was revealed that the amount of Enterobacteriaceae was increased via the administration of an extract containing resveratrol (Giuliani et al., 2016). Therefore, resveratrol could have a regulative effect on gut microbiota. Thus, the effect of resveratrol on gut microbial modulation will be discussed (Tables 1 and 2).

Using combinations of phytochemicals in disease amelioration has become a trend, as doing so follows the concept of precise prevention. Additionally, different compounds may not share the same targets. Early in 2015, it was revealed that, compared to quercetin, trans-resveratrol could scarcely modify the gut bacteria profile and more likely contributes to the mRNA expression of tight-junction related proteins and inflammation-associated genes (Etxeberria et al., 2015). As phytochemicals with low bioavailability, resveratrol and quercetin surprisingly showed distinct effects on gut health. Yang et al. demonstrated that resveratrol showed a positive effect on controlling blood glucose and increasing HDL-c levels, while sinapic acid exhibited alleviative effects on oxidative stress, TGs (triglycerides), and NEFAs (non-esterified fatty acids) in plasma. The authors revealed that a combination of the two could suppress the growth of disease-related species, such as Bacteroides and Desulfovibrionaceae $s p$, and increase the abundance of the genera Blautia and Dorea from the family Lachnospiraceae (Yang et al., 2019). These results showed that combinations of phytochemicals could have beneficial or even synergistic effects on disease prevention. However, Sreng et al. suggested that the glycemic regulative effect of resveratrol could be blunted by curcumin, thus indicating that it is possible for a phytochemical to have an antagonistic ef- 


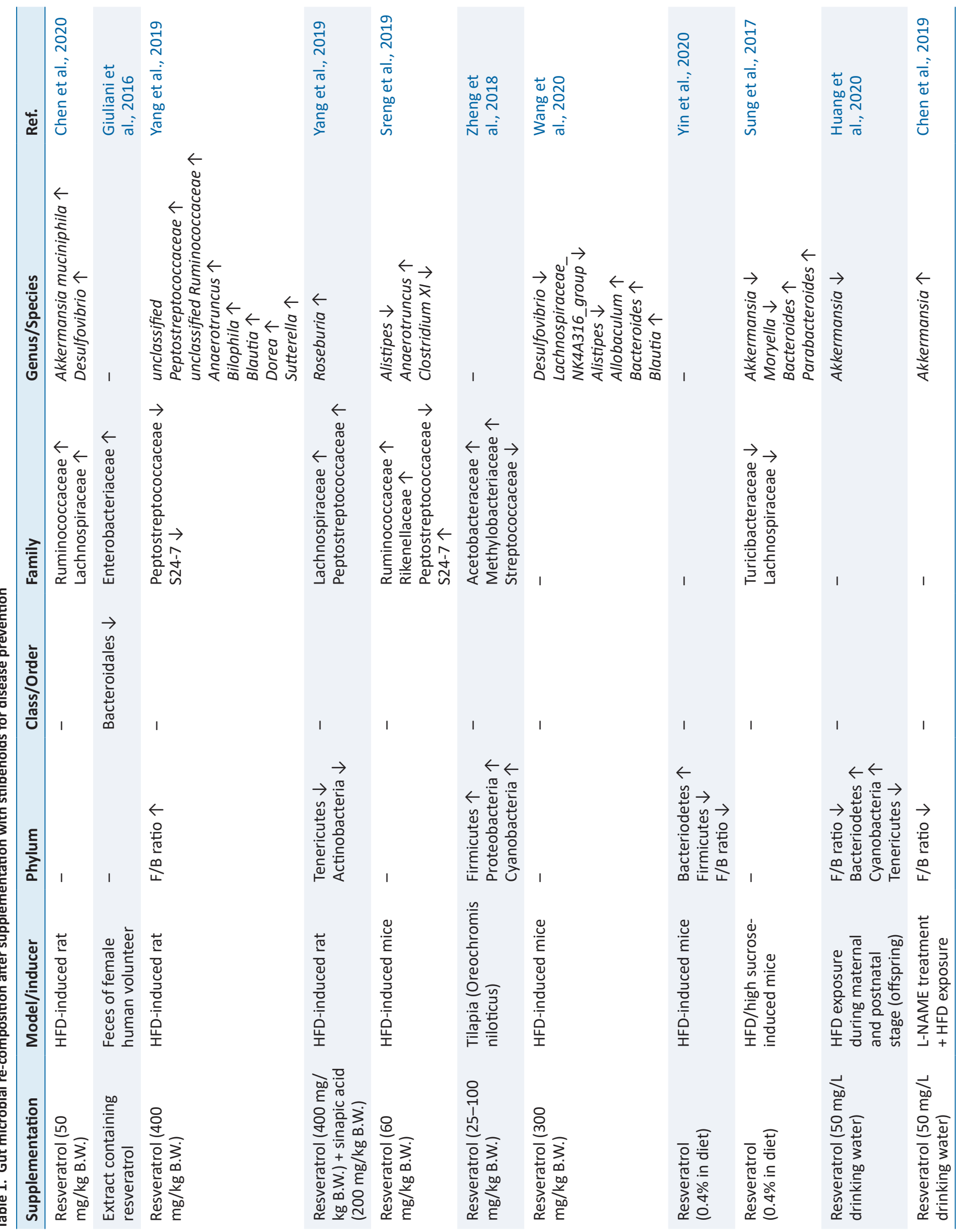




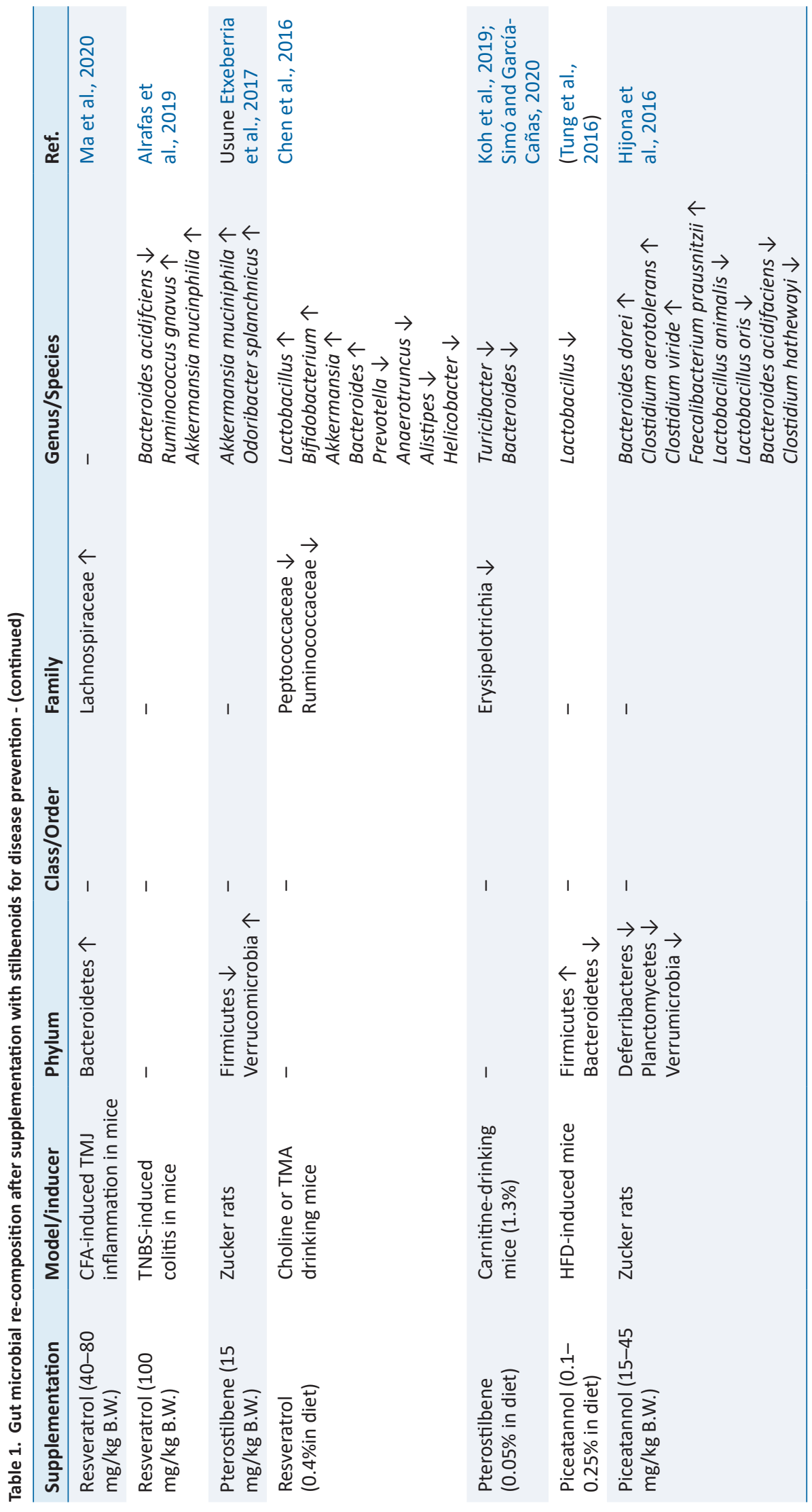




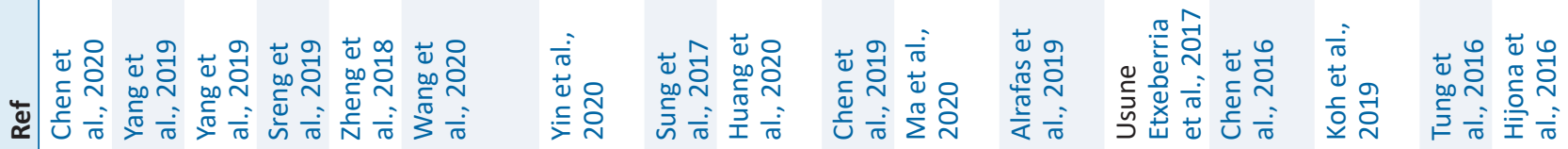
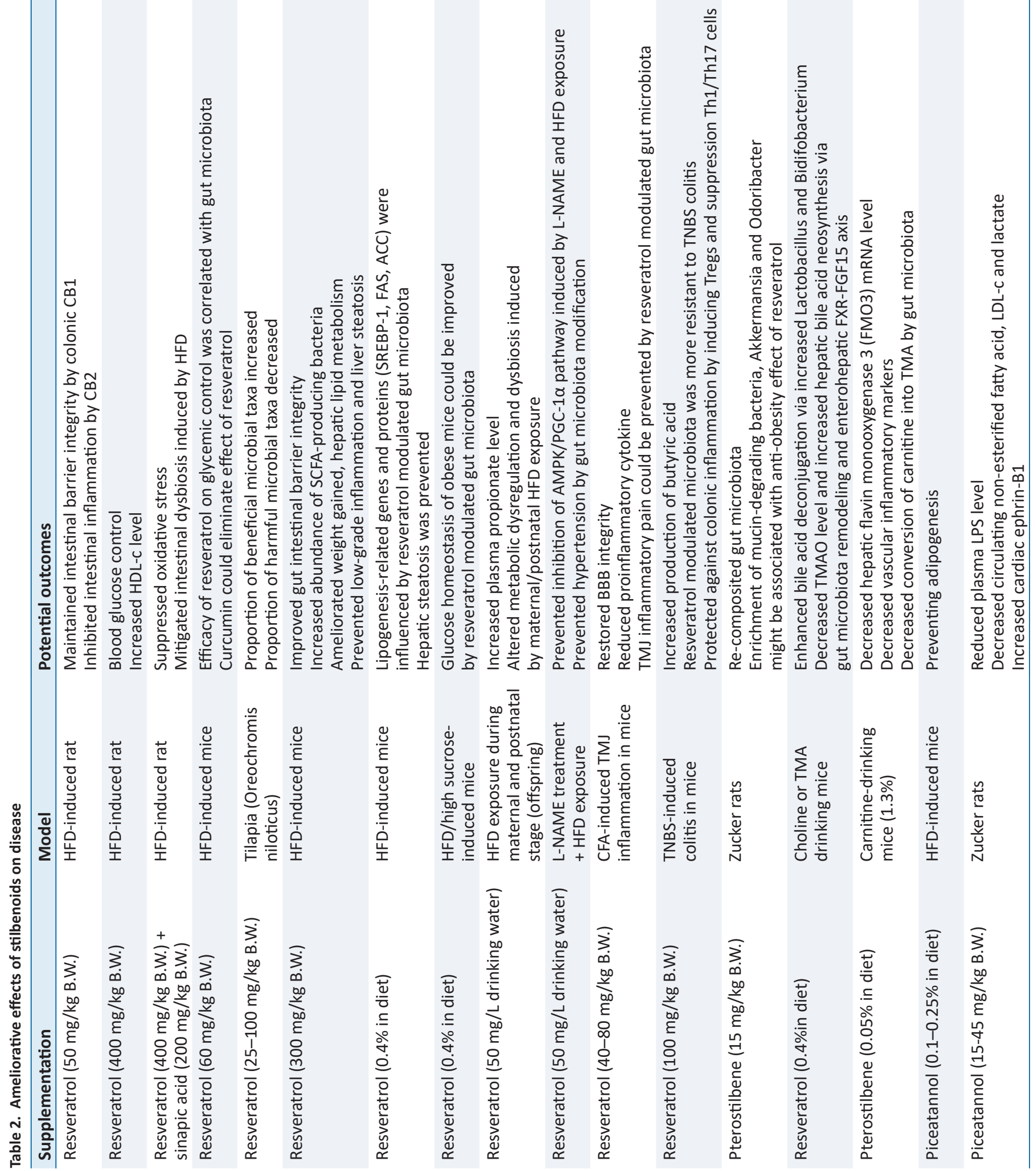
fect. In gut microbiota analyses, it has been suggested that some bacterial taxons correlate to the glycemic index, including S477 (family) and Lactobacillus and Prevotella (genes), which have negative correlations, as well as Peptococcaceae (family) and $\mathrm{Mu}$ cisporollum schaedleri, which positively correlate with the glycemic index. In comparison, both resveratrol and curcumin alone led to changes in microbial composition. However, the reversed effect of resveratrol, in terms of reducing Alistipes and Clostridium XI was masked when combining with curcumin (Sreng et al., 2019). Therefore, care should be taken in combining phytochemicals as a strategy for disease prevention.

Zheng et al. suggested that dietary supplementation with resveratrol significantly enriched bacteria of the phylum Firmicutes in farmed tilapia (Zheng et al., 2018). Moreover, the resveratrol supplemented microbiota were dominated by Proteobacteria, Firmicutes, and Cyanobacteria, with increases in Acetobacteraceae and Methylobacteriaceae and reductions in Streptococcaceae. In another study, the high-fat diet (HFD)-induced nonalcoholic fatty liver disease (NAFLD) in a mouse model suggests that supplementation with resveratrol could modulate gut microbial composition, including decreasing Lachnospiraceae_NK4A316_group, Alistipis, and Desulfovibrio concomitant with increases in the abundance of SCFA-producing bacteria, namely, Allovaculum, Bacteroides, and Blautia (Wang et al., 2020). Moreover, transplantation of the fecal microbiota of resveratrol-supplemented mice into HFD-induced mice could significantly prevent obesity, NAFLD, and inflammation, indicating that resveratrol-modified gut microbiota could potentially prevent obesity-related diseases induced by HFD. A study by Yin et al. obtained a similar result in that it revealed that transplantation of fecal microbiota from mice fed a chow diet supplemented with resveratrol could both ameliorate the hepatic steatosis and reverse the gut microbiota dysbiosis in mice fed a HFD (Yin et al., 2020). The Firmicutes/Bacteriodetes (F/B) ratio of the fecal microbiota for FMT (fecal microbiota transplant) was reversed and showed no significant difference from the ratios for mice fed a chow diet or a chow diet supplemented with resveratrol. In a $\mathrm{HFD} /$ high sucrose induced diabetes model in mice, it was revealed that obesity and glucose homeostasis dysregulation was ameliorated by oral administration of resveratrol, and it might be related to decreases in Turicibacteraceae, Lachnospiraceae, Moryella, and Akkermansia and increases in Bacteroides and Parabacteroides (Sung et al., 2017). The FMT model was also employed to confirm that gut microbiota from resveratrol-supplemented donors could improve glucose homeostasis of mice.

In summary, a HFD can lead to gut microbial dysbiosis and a deterioration in the host's metabolism. In 2020, Huang et al. revealed that resveratrol was capable of improving the microbialdysbiosis-damaged metabolisms of offspring induced by HFD exposure during maternal and postnatal diet intake (Huang et al., 2020). In terms of the SCFA levels, maternal and postnatal HFD exposure led to reductions in the plasma SCFA concentration, while supplementation with resveratrol increased propionate levels in offspring in both situations. In the microbial analysis, it was found that lactate-producing bacteria had decreased in the early life of offspring due to maternal exposure to HFD. In adulthood, both maternal and postnatal HFD exposure sharply increased the F/B ratio (up to 10-fold) compared to a control group. Fortunately, the changes were reversed by supplementation with resveratrol, which was reflected in the increases in Bacteroidetes and Cyanobacteria and decreases in Tenericutes. The modulation of resveratrol also occurred at the genus level, as indicated by the decrease in the Akkermansia level. In 2019, the same research team demonstrated that resveratrol exhibited a preventive effect on programmed hypertension and gut microbiota (Chen et al., 2019). In the study, treatment with L-NAME (NG-nitro-L-arginine-methyl ester) combined with a HFD led to hypertension and an increased F/B ratio. However, supplementation with resveratrol prevented both outcomes as well increased Akkermansia, which is from the phylum Verrucomicrobia. Therefore, resveratrol had a positive impact on gut microbiota regulation, which altered metabolic dysregulation and lowered hypertension.

In addition to metabolic syndromes, other diseases may be correlated to gut microbial composition. For instance, temporomandibular disorder, a neurological disease that results in chronic pain, was found to have a possible pathogenesis caused by disturbances in the gut microbiota (Ma et al., 2020). In the study, a complete Freund's adjuvant was injected at the intra-temporomandibular joint, and a reduction in SCFA production was observed. Surprisingly, the disturbance was diminished by resveratrol, concomitant with increased SCFA levels. In the study of 2,4,6-trinitrobenzenesulfonic acid (TNBS)-induced colitis in mice, resveratrol not only attenuated colonic inflammation but the homeostatic levels of gut microbes recovered (Hijona et al., 2016). Additionally, bacteroides acidfaciens induced by TNBS was significantly reduced, while Ruminococcus gnavus and Akkermansia muciniphila were significantly induced, concomitant with acetic and butyric acid concentrations (Alrafas et al., 2019).

As a phytochemical with low bioavailability, the reason as to why resveratrol is capable of effectively ameliorating several diseases in the body has not been clearly elucidated. However, it is believed that the modification of the gut microbial composition in recent studies could supply a partial reason. Changes in gut microbes via resveratrol supplementation may lead to potential benefits to the host in disease amelioration, especially in terms of obesity-related syndromes. Nevertheless, the underlying details still need to be clarified.

\section{The role of pterostilbene in modulating gut microbiota to produce a host health benefit}

Compared to resveratrol, the bioavailability of pterostilbene (Figure 1) is much higher, but complete absorption is impossible. Therefore, the interaction between pterostilbene and gut microbiota should not be overlooked. However, studies focusing on interactions between pterostilbene and gut microbiota have not been as well-clarified as those involving resveratrol. Therefore, in this section, the anti-microbial effects of pterostilbene will be discussed to postulate the potential of pterostilbene in gut microbial modulation (Tables 1 and 2).

In a study involving Zucker rats fed either a standard diet or a pterostilbene-supplemented diet, it was found that pterostilbene not only exerted anti-obesity effects but also re-structured gut microbial composition (Etxeberria et al., 2017). In particular, a reduction in the abundance of Firmicutes and an increase in the phylum Verrucomicrobia was observed. Akkermandia muciniphila and Odoribacter splanchnicus (members of Verrucomicrobia and Bacteroidetes, respectively) were found to increase in abundance in the supplemented group. The former was found to be inversely correlated with obesity, while the latter was negatively correlated with adiposity. In addition to obesity, the incidence of cardiovascular disease is also highly related to the composition of gut microbiota, as the role of gut microbiota in the conversion of carnitine to trimethylamine has been clarified recent years (Simó and García-Cañas, 2020). In the aforementioned study, the abundance of Turicibacter from Erysipelotrichia (family) was significantly increased in the carnitine group, while pterostilbene supplementa- 
<smiles>Oc1ccc(/C=C/c2cc(O)cc(O)c2)cc1</smiles>

b<smiles>Oc1ccc(CCc2cc(O)cc(O)c2)cc1</smiles>

C<smiles>Oc1ccc(/C=C/c2cccc(O)c2)cc1</smiles>

d<smiles>Oc1ccc(CCc2cccc(O)c2)cc1</smiles>

e<smiles>Oc1cccc(/C=C/c2cc(O)cc(O)c2)c1</smiles><smiles>Oc1cccc(/C=C/c2ccc(O)c(O)c2)c1</smiles>

g<smiles>Oc1cccc(CCc2ccc(O)c(O)c2)c1</smiles>

Figure 2. Potential metabolites or derivatives of the stilbenoids depicted in Figure 1 formed via gut microbial transformation. (a) Resveratrol, natural compound provided for structural comparison; (b) dihydroresveratrol; (c) 3,4'-dihydroxy-trans-stilbene; (d) lunularin, (e) isoresveratrol; (f) 3,3',4'-trihydroxystilbene or 3',4',5-trihydroxystilbene; and (g) 3,3',4'-trihydroxybibenzyl.

tion led to its reduction and increased Bacteroides. In comparison, resveratrol exhibited a similar effect, mainly on gut microbiota remodeling to prevent the production of trimethylamine-N-oxide (TMAO) from trimethylamine (TMA) (Chen et al., 2016). The increased abundance of Lactobacillus and Bifidobacterium might be closely related to bile salt hydrolase activity and bile acid deconjugation.

The secretion of stilbenoids occurs naturally in some plants as protection against infections; therefore, most of them could exhibit anti-microbial effects (Akinwumi et al., 2018). Inhibiting the growth of pathogenic or disease-related microbes is one of the strategies used in disease prevention. In fact, the antibacterial and antifungal properties of resveratrol have been reviewed by Vestergaard and Ingmer (Vestergaard and Ingmer, 2019). Therefore, the inhibition of adverse gut microbial growth could be a potential strategy in gut microbial re-composition. Mattio et al. suggested that among resveratrol and its derived monomers, pterostilbene showed significant anti-bacterial capabilities against gram-positive foodborne pathogens (Mattio et al., 2019). In 2017, Yang et al. demonstrated that pterostilbene exhibited anti-bacterial effects against Staphylococcus aureus, which is resistant to methicillin, and was even more efficient than resveratrol (Yang et al., 2017). The capability of Staphylococcus aureus to form biofilms was eradicated by pterostilbene, thus highly reducing its resistance to antibiotics and immune cells. In 2020, a similar result was observed in that pterostilbene was able to inhibit the growth and formation of Staphylococcus epidermidis, and more importantly, the pterostilbene-enhanced anti-microbial activity of antibiotics, including erythromycin and tetracycline (Kašparová et al., 2020). The enhanced effects of pterostilbene on antibiotics were also observed in combination with gentamicin, as they synergistically inhibited Staphylococcus aureus ATCC 25923, Escherichia coli O157, and Pseudomonas aeruginosa 15442 (Lee et al., 2017).

Supplementation of pterostilbene is not as well-studied as sup- plementation with resveratrol. However, pterostilbene's anti-microbial abilities indicate its high potential to prevent pathogenic microbial diseases and inhibit the growth of adverse gut microbes, resulting in gut health improvement. Therefore, more research is required to ensure its beneficial properties related to the gut.

4. The role of gut microbiota in the conversion of stilbenoids in terms of their bioactivity

As mentioned above, it is believed that interactions between phytochemicals and gut microbiota may reflect metabolite production, derivatives (Figures 1 and 2), and gut microbial composition. In a moderate long-term (28-day) consumption of red wine or dealcoholized red wine study involving humans, up to 21 metabolites of resveratrol were identified in urine, and it was suggested that some of them were gut microbial-derived metabolites, although no further analyses took place (Rotches-Ribalta et al., 2012). To be more specific, dihydroresveratrol was the most prevalent gut microbial metabolite, followed by 3,4'-dihydroxy-trans-stilbene and lunularin. Dihydroresveratrol was found to be produced by Slackia equolifaciens and Adlercreutzia equolifaciens, while the production of lunularin was related to the increased abundance of Bacteroidetes, Actinobacter, Verrucomicrobia, and Cyanobacteria (Bode et al., 2013).

In 2019, Jarosova et al. observed the metabolic transformation of six stilbenoids (resveratrol, oxyresveratrol, piceatannol, thunalbene, batatasin III, and pinostilbene) by using a fecal fermentation system, with fresh feces supplied by five donors (Jarosova et al., 2019). Among the selected stilbenoids, the following conversions were observed: resveratrol into dihydroresveratrol, oxyresveratrol into 2'3,4',5-tetrahydroxybibenzyl, and thunalbene into isoresveratrol. The metabolites of piceatannol found in this in vitro study included dihydropiceatannol, and 3,3'4'-trihydroxystilbene, or 
3'4'5-trihydroxystilbene, and the latter compounds could be further converted into 3,3',4'-trihydroxybibenzyl. Note that batatasin III and pinostilbene might be too stable to be transformed further in a colon environment. In another animal study, it was suggested that intragastric administration of resveratrol and piceatannol in rats not only leads to conjugated metabolites but also the methylation of piceatannol to form $O$-methyl piceatannol and its conjugates (Setoguchi et al., 2014).

In addition to conversions between stilbenoid monomers, since 1998 , there have been several studies that have mentioned the oligomerization of resveratrol and pterostilbene to form dimers or trimers through catalyzation with laccase (Jeandet et al., 2020). Nevertheless, although the microbial cultures contributing the laccase were not bacterial strains, they still provided the potential to think in terms of stilbenoid metabolism.

\section{Interactions between gut microbiota and analogues of res- veratrol and pterostilbene}

Resveratrol is the most well-studied member of the stilbenoid group due to its marvelous health benefits (Table 1). Therefore, its analogues have gained attention for their potential in disease prevention (Table 2). However, only a few studies have mentioned their effects on gut microbiota modulation, which, in turn, also leads to health benefits. Hereinafter, some of the reported stilbenoids with benefits in terms of disease amelioration via interactions with gut microbiota will be discussed.

Piceatannol (Figure 1) is a hydroxyl derivative of resveratrol that is naturally derived from some plants, such as Euphorbia lagascae, and some of its beneficial properties have been reported previously (Osman et al., 2016). In 2016, it was revealed that the anti-obesity effects of piceatannol may be partially attributed to the re-composition of gut microbiota (Tung et al., 2016). Briefly, piceatannol increased the relative abundance of Firmicutes, while it decreased Bacteroidetes and Lactobacillus, as compared to the group given a HFD without piceatannol supplementation. Another study supplemented diets given to Zucker rats with piceatannol and found that the effect was not as expected; however, some changes were observed in terms of the relative abundance of fecal microbiota. In particular, although piceatannol did not lead to many significant changes in phylums, it did significantly reduce Lactobacillus animalis, Lactobacillus oris, Bacteroides acidifaciens, and Clostidium hathewayi and led to increases in Bacteroides dorei, Clostidium aerotolerans, Clostidium viride, and Faecalibacterium prausnitzii (Hijona et al., 2016).

In another in vitro study, intervention with stilbenoids (resveratrol, oxyresveratrol, batatasin III, piceatannol, pinostilbene, and thunalbene) was found to modulate the microbial compositions originating from four donors (Jaimes et al., 2019). In addition to a decrease in the $\mathrm{F} / \mathrm{B}$ ratio, there was a reduction in Clostridium and elevations in Faecalibacterium prausnitzii and Ruminococcus gnavus. Notably, the abundance of Faecalibacterium prausnitzii was suggested to be related to the production of SCFAs. In comparison, resveratrol and piceatannol exhibited the greatest effects on the modulation of microbial composition, followed by thunalbene and batatasin III.

\section{Future perspectives}

In summary, the regulative capability of resveratrol on gut microbiota has been well-defined and partially contributes to the preven- tion of several diseases. The analogues of resveratrol, including pterostilbene and piceatannol, are interesting compounds that also lead to changes in gut microbial composition. Notably, pterostilbene has a synergistic effect when combined with different antibiotics. However, the role of stilbenoids in disease alleviation should be further clarified. In addition, the transformations of individual stilbenoids in the colon environment, either via microbial conversion or colonic/hepatic enzymatic transformation or conjugation, also need to be further investigated. The bioactive effect of these metabolites should not be neglected, as some of them have been proven to have disease prevention capabilities in recent studies. All in all, stilbenoids may be striking choices for development as chemopreventive agents, and most importantly, their modulatory effects on gut microbial may greatly increase their potential for improving gut health.

\section{References}

Akinwumi, B.C., Bordun, K.-A.M., and Anderson, H.D. (2018). Biological Activities of Stilbenoids. Int. J. Mol. Sci. 19: 792.

Alrafas, H.R., Busbee, P.B., Nagarkatti, M., and Nagarkatti, P.S. (2019). Resveratrol modulates the gut microbiota to prevent murine colitis development through induction of Tregs and suppression of Th17 cells. J Leukoc Biol. 106: 467-480.

Bode, L.M., Bunzel, D., Huch, M., Cho, G.-S., Ruhland, D., Bunzel, M., Bub, A., Franz, C.M.A.P., and Kulling, S.E. (2013). In vivo and in vitro metabolism of trans-resveratrol by human gut microbiota. Am J Clin Nutr. 97: 295-309.

Carrera-Quintanar, L., López Roa, R.I., Quintero-Fabián, S., SánchezSánchez, M.A., Vizmanos, B., and Ortuño-Sahagún, D. (2018). Phytochemicals That Influence Gut Microbiota as Prophylactics and for the Treatment of Obesity and Inflammatory Diseases. Mediators Inflamm. 2018: 9734845

Chen, H.-E., Lin, Y.-J., Lin, I.C., Yu, H.-R., Sheen, J.-M., Tsai, C.-C., Huang, L.-T., and Tain, Y.-L. (2019). Resveratrol prevents combined prenatal NG-nitro-L-arginine-methyl ester (L-NAME) treatment plus postnatal high-fat diet induced programmed hypertension in adult rat offspring: interplay between nutrient-sensing signals, oxidative stress and gut microbiota. J. Nutr. Biochem. 70: 28-37.

Chen, M., Hou, P., Zhou, M., Ren, Q., Wang, X., Huang, L., Hui, S., Yi, L., and $\mathrm{Mi}, \mathrm{M}$. (2020). Resveratrol attenuates high-fat diet-induced non-alcoholic steatohepatitis by maintaining gut barrier integrity and inhibiting gut inflammation through regulation of the endocannabinoid system. Clin Nutr. 39: 1264-1275.

Chen, M.-L., Yi, L., Zhang, Y., Zhou, X., Ran, L., Yang, J., Zhu, J.-d., Zhang, Q.-y., and Mi, M.-t. (2016). Resveratrol Attenuates Trimethylamine-NOxide (TMAO)-Induced Atherosclerosis by Regulating TMAO Synthesis and Bile Acid Metabolism via Remodeling of the Gut Microbiota. mBio. 7: e02210-e02215.

Etxeberria, U., Arias, N., Boqué, N., Macarulla, M.T., Portillo, M.P., Martínez, J.A., and Milagro, F.I. (2015). Reshaping faecal gut microbiota composition by the intake of trans-resveratrol and quercetin in highfat sucrose diet-fed rats. J. Nutr. Biochem. 26: 651-660.

Etxeberria, U., Hijona, E., Aguirre, L., Milagro, F.I., Bujanda, L., Rimando, A.M., Martínez, J.A., and Portillo, M.P. (2017). Pterostilbene-induced changes in gut microbiota composition in relation to obesity. Mol. Nutr. Food Res. 61: 1500906

Giuliani, C., Marzorati, M., Innocenti, M., Vilchez-Vargas, R., Vital, M., Pieper, D.H., Van de Wiele, T., and Mulinacci, N. (2016). Dietary supplement based on stilbenes: a focus on gut microbial metabolism by the in vitro simulator M-SHIME ${ }^{\circledR}$. Food Funct. 7: 4564-4575.

Hijona, E., Aguirre, L., Pérez-Matute, P., Villanueva-Millán, M.J., MosquedaSolis, A., Hasnaoui, M., Nepveu, F., Senard, J.M., Bujanda, L., AldámizEchevarría, L., Llarena, M., Andrade, F., Perio, P., Leboulanger, F., Hijona, L., Arbones-Mainar, J.M., Portillo, M.P., and Carpéné, C. (2016). Limited beneficial effects of piceatannol supplementation on obesity complications in the obese Zucker rat: gut microbiota, metabolic, endocrine, and cardiac aspects. J. Physiol. Biochem. 72: 567-582. 
Hinderliter, P., and Saghir, S.A. (2014). Encyclopedia of Toxicology (Third Edition). Academic Press, Oxford, pp. 849-855.

Huang, Y.-C., Huang, L.-T., Sheen, J.-M., Hou, C.-Y., Yeh, Y.-T., Chiang, C.-P., Lin, I.C., Tiao, M.-M., Tsai, C.-C., Lin, Y.-J., Chen, C.-C., Tain, Y.-L., and Yu, H.-R. (2020). Resveratrol treatment improves the altered metabolism and related dysbiosis of gut programed by prenatal high-fat diet and postnatal high-fat diet exposure. J. Nutr. Biochem. 75: 108260.

Jaimes, J.D., Jarosova, V., Vesely, O., Mekadim, C., Mrazek, J., Marsik, P., Killer, J., Smejkal, K., Kloucek, P., and Havlik, J. (2019). Effect of Selected Stilbenoids on Human Fecal Microbiota. Molecules. 24: 744.

Jarosova, V., Vesely, O., Marsik, P., Jaimes, J.D., Smejkal, K., Kloucek, P., and Havlik, J. (2019). Metabolism of Stilbenoids by Human Faecal Microbiota. Molecules. 24: 1155

Jeandet, P., Sobarzo-Sánchez, E., Silva, A.S., Clément, C., Nabavi, S.F., Battino, M., Rasekhian, M., Belwal, T., Habtemariam, S., Koffas, M., and Nabavi, S.M. (2020). Whole-cell biocatalytic, enzymatic and green chemistry methods for the production of resveratrol and its derivatives. Biotechnol. Adv. 39: 107461.

Kašparová, P., Vaňková, E., Brázdová, L., Lokočová, K., Matátková, O., and Masák, J. (2020). Antibiofilm agent pterostilbene is able to enhance antibiotics action against Staphylococcus epidermidis. Microb. Pathog 104632In Press.

Koh, Y.-C., Li, S., Chen, P.-Y., Wu, J.-C., Kalyanam, N., Ho, C.-T., and Pan, M.H. (2019). Prevention of Vascular Inflammation by Pterostilbene via Trimethylamine-N-Oxide Reduction and Mechanism of Microbiota Regulation. Mol. Nutr. Food Res. 63: 1900514

Lee, W.X., Basri, D.F., and Ghazali, A.R. (2017). Bactericidal Effect of Pterostilbene Alone and in Combination with Gentamicin against Human Pathogenic Bacteria. Molecules. 22: 463.

Ma, Y., Liu, S., Shu, H., Crawford, J., Xing, Y., and Tao, F. (2020). Resveratrol alleviates temporomandibular joint inflammatory pain by recovering disturbed gut microbiota. Brain Behav. Immun. 87: 455-464.

Manning, T.S., and Gibson, G.R. (2004). Prebiotics. Best Pract Res Clin Gastroenterol. 18: 287-298.

Martel, J., Ojcius, D.M., Ko, Y.-F., and Young, J.D. (2020). Phytochemicals as Prebiotics and Biological Stress Inducers. Trends Biochem. Sci. 45 462-471.

Mattio, L.M., Dallavalle, S., Musso, L., Filardi, R., Franzetti, L., Pellegrino, L., D'Incecco, P., Mora, D., Pinto, A., and Arioli, S. (2019). Antimicrobia activity of resveratrol-derived monomers and dimers against foodborne pathogens. Sci. Rep. 9: 19525.

Nikinmaa, M. (2014). Chapter 6 - Factors Affecting the Bioavailability of Chemicals. An Introduction to Aquatic Toxicology. Academic Press, Oxford, pp. 65-72.

Osman, A.G., Chittiboyina, A.G., and Khan, I.A. (2016). Chapter Three - Cytoprotective Role of Dietary Phytochemicals Against Cancer Development via Induction of Phase II and Antioxidant Enzymes. Advances in Molecular Toxicology. Elsevier, pp. 99-137.

Peng, M., Tabashsum, Z., Anderson, M., Truong, A., Houser, A.K., Padilla, J., Akmel, A., Bhatti, J., Rahaman, S.O., and Biswas, D. (2020). Effectiveness of probiotics, prebiotics, and prebiotic-like components in common functional foods. Compr. Rev. Food Sci. Food Saf. 19: 1908-1933.

Rotches-Ribalta, M., Urpi-Sarda, M., Llorach, R., Boto-Ordoñez, M., Jauregui, O., Chiva-Blanch, G., Perez-Garcia, L., Jaeger, W., Guillen, M., Corella, D., Tinahones, F.J., Estruch, R., and Andres-Lacueva, C. (2012). Gut and microbial resveratrol metabolite profiling after moderate long-term consumption of red wine versus dealcoholized red wine in humans by an optimized ultra-high-pressure liquid chromatography tandem mass spectrometry method. J. Chromatogr. A. 1265: 105-113.

Selby-Pham, S.N.B., Miller, R.B., Howell, K., Dunshea, F., and Bennett, L.E. (2017). Physicochemical properties of dietary phytochemicals can predict their passive absorption in the human small intestine. Sci. Rep. 7: 1931.

Setoguchi, Y., Oritani, Y., Ito, R., Inagaki, H., Maruki-Uchida, H., Ichiyanagi, T., and Ito, T. (2014). Absorption and Metabolism of Piceatannol in Rats. J. Agric. Food Chem. 62: 2541-2548.

Simó, C., and García-Cañas, V. (2020). Dietary bioactive ingredients to modulate the gut microbiota-derived metabolite TMAO. New opportunities for functional food development. Food Funct. 11: 67456776

Sreng, N., Champion, S., Martin, J.-C., Khelaifia, S., Christensen, J.E., Padmanabhan, R., Azalbert, V., Blasco-Baque, V., Loubieres, P., Pechere, L., Landrier, J.-F., Burcelin, R., and Sérée, E. (2019). Resveratrol-mediated glycemic regulation is blunted by curcumin and is associated to modulation of gut microbiota. J. Nutr. Biochem. 72: 108218.

Sung, M.M., Kim, T.T., Denou, E., Soltys, C.-L.M., Hamza, S.M., Byrne, N.J. Masson, G., Park, H., Wishart, D.S., Madsen, K.L., Schertzer, J.D., and Dyck, J.R.B. (2017). Improved Glucose Homeostasis in Obese Mice Treated With Resveratrol Is Associated With Alterations in the Gut Microbiome. Diabetes. 66: 418.

Tung, Y.-C., Lin, Y.-H., Chen, H.-J., Chou, S.-C., Cheng, A.-C., Kalyanam, N., Ho, C.-T., and Pan, M.-H. (2016). Piceatannol Exerts Anti-Obesity Effects in C57BL/6 Mice through Modulating Adipogenic Proteins and Gut Microbiota. Molecules. 21: 1419.

Vestergaard, M., and Ingmer, H. (2019). Antibacterial and antifungal properties of resveratrol. Int. J. Antimicrob. Agents. 53: 716-723.

Waller, D.G., and Sampson, A.P. (2018). Chapter 2 - Pharmacokinetics. Medical Pharmacology and Therapeutics (Fifth Edition). Elsevier, pp. 33-62.

Wang, P., Wang, J., Li, D., Ke, W., Chen, F., and Hu, X. (2020). Targeting the gut microbiota with resveratrol: a demonstration of novel evidence for the management of hepatic steatosis. J. Nutr. Biochem. 81 108363.

Yang, C., Deng, Q., Xu, J., Wang, X., Hu, C., Tang, H., and Huang, F. (2019). Sinapic acid and resveratrol alleviate oxidative stress with modulation of gut microbiota in high-fat diet-fed rats. Int. Food Res. J. 116: 1202-1211.

Yang, S.-C., Tseng, C.-H., Wang, P.-W., Lu, P.-L., Weng, Y.-H., Yen, F.-L., and Fang, J.-Y. (2017). Pterostilbene, a Methoxylated Resveratrol Derivative, Efficiently Eradicates Planktonic, Biofilm, and Intracellular MRSA by Topical Application. Front Microbiol. 8: 1103.

Yin, X., Liao, W., Li, Q., Zhang, H., Liu, Z., Zheng, X., Zheng, L., and Feng, X. (2020). Interactions between resveratrol and gut microbiota affect the development of hepatic steatosis: A fecal microbiota transplantation study in high-fat diet mice. J. Funct. Foods. 67: 103883.

Zheng, Y., Wu, W., Hu, G., Qiu, L., Meng, S., Song, C., Fan, L., Zhao, Z., Bing, X., and Chen, J. (2018). Gut microbiota analysis of juvenile genetically improved farmed tilapia (Oreochromis niloticus) by dietary supplementation of different resveratrol concentrations. Fish Shellfish Immunol. 77: 200-207. 